\title{
Mastoid Obliteration Using Bioactive Glass-Our Experience
}

\author{
Anand Velusamy ${ }^{1} \quad$ Nazrin Hameed ${ }^{1} \quad$ Aishwarya Anand ${ }^{1}$ \\ ${ }^{1}$ Department of Otorhinolaryngology, Muthur Chinnasamy \\ Velusamy Ear Nose and Throat Centre, Coimbatore, Tamil Nadu, \\ India \\ Address for correspondence Nazrin Hameed, MBBS, MS, DNB, \\ MRCS, Department of Otorhinolaryngology, MCV ENT Centre, \\ Coimbatore- 641045, Tamil Nadu, India \\ (e-mail:drnazrinent@gmail.com).
}

Ann Otol Neurotol ISO 2021;4:57-61.

\begin{abstract}
Keywords

- cholesteatoma

- mastoid obliteration

- bioglass

Aims The aim of this study was to evaluate the surgical outcome of cavity obliteration with bioactive glass in patients with cholesteatoma undergoing canal wall down mastoidectomy with reconstruction of the canal wall.

Materials and Methods A prospective study was conducted over a period of 3 years on 25 patients who underwent mastoid obliteration with bioactive glass following canal wall down mastoidectomy for cholesteatoma. The primary outcome measure was the presence of a dry, low-maintenance mastoid cavity that was free of infection, assessed, and graded according to the grading system by Merchant et al at the end of 1 and 6 months postoperatively. Secondary outcome measures included presence of postoperative complications like wound infection, posterior canal wall bulge, and residual perforation.

Results Out of the 25 patients on whom this study was conducted, at the end of 1 month $60 \%$ had a completely dry ear, $28 \%$ of patients had grade 1 , and $12 \%$ had grade 2 otorrhea at the end of the first month. At the end of 6 months, $72 \%$ had a completely dry ear, while $20 \%$ had grade 1 and $8 \%$ had grade 2 otorrhea. There were no cases with grade 3 otorrhea during the entire follow-up period. Postoperative complications of the posterior canal bulge were noted in two patients (8\%), and one patient (4\%) had a residual perforation.

Conclusion Mastoid cavity obliteration with bioactive glass is an effective technique to avoid cavity problems.
\end{abstract}

\section{Introduction}

In 1911, Mosher introduced the concept of mastoid obliteration to facilitate healing process in mastoidectomy cavity. He originally described a postauricular soft tissue flap that was superiorly based. ${ }^{1}$ Following that numerous other techniques have been reported over the past many decades, which include local flaps (muscle, periosteum, fascia), free grafts (bone, cartilage, etc.), and synthetic materials (hydroxyapatite, Plastipore). Reconstruction of posterior canal wall

Published online

September 16, 2021
DOI https://doi.org/

$10.1055 / \mathrm{s}-0041-1735414$

ISSN 2581-9607 with tragal, conchal cartilage, and septal cartilage has been tried. However, all of the techniques have advantages and disadvantages.

Mastoid obliteration is most commonly required after a canal wall down mastoidectomy for chronic otitis media with cholesteatoma. A canal wall down mastoid cavity, if not obliterated, can result in the cavity problems such as persistent otorrhea, need for frequent cleaning, difficulty to use hearing aid, and tendency to evoke vertigo due to caloric stimulus such as warm or cold air or water. In such cases, the otorrhea

(C) 2021. Indian Society of Otology.

This is an open access article published by Thieme under the terms of the Creative Commons Attribution-NonDerivative-NonCommercial-License, permitting copying and reproduction so long as the original work is given appropriate credit. Contents may not be used for commercial purposes, or adapted, remixed, transformed or built upon. (https://creativecommons.org/licenses/by-nc-nd/4.0/).

Thieme Medical and Scientific Publishers Pvt. Ltd. A-12, 2nd Floor, Sector 2, Noida-201301 UP, India 
may fail to respond to antibiotic therapy and frequent aural toilet. Reducing the cavity problems by reduction in size of cavity is the primary purpose of performing a mastoid cavity obliteration and it is usually done as a primary procedure during canal wall down mastoidectomies. ${ }^{2}$

Obliteration of the mastoid cavity promotes quicker healing by reducing the surface area requiring epithelialization. Small cavity is also more likely to retain its epithelial migratory potential and is self-cleaning. Following mastoidectomy, the exposed bone will leak transudate, which acts as a rich medium for microbial growth. By covering the bony walls, we can limit the process of transudation thereby reducing the infection risk. ${ }^{3}$

Bioactive glass (BAG) is a newer biocompatible material with a wide spectrum of important properties such as its antibacterial effect, nontoxic property, and also induction of new bone formation. ${ }^{4}$ BAG has been used in reconstruction of defects of facial bones and in areas potentially contaminated with microbial infectious, such as in frontal sinusitis and repair of nasal septal perforations..$^{5-8}$

\section{Materials and Methods}

The aim of this study was to evaluate the surgical outcome of cavity obliteration with BAG in cases of cholesteatoma undergoing canal wall down mastoidectomy with reconstruction of the canal wall.

A prospective study was conducted over a period of 3 years at MCV ENT Hospital, Pollachi, including 25 patients with cholesteatoma who underwent canal wall down mastoidectomy with reconstruction of the canal wall and patients with cavity problems in the age group between 11 and 60 years. Following confirmation of diagnosis of cholesteatoma obtained by detailed medical history, clinical evaluation and radiological evaluation patients were taken up for surgery.

Patients were made to lie in supine position with the affected ear under microscope. Under aseptic precautions, local infiltration was given in the postaural region following which a postauricular skin crease incision was made and dissected, thereby developing a plane between the subcutaneous tissue and temporalis muscle and periosteum of the mastoid. Temporalis fascia graft was harvested and set aside to dry. Following postaural soft tissue dissection and periosteal elevation, the mastoid cortex was exposed and an intact skin tube flap was elevated and secured anteriorly. A simple mastoidectomy was done and cholesteatoma and granulations filling the central mastoid tracts were removed at this time. Posterior canal wall was safely thinned out and canal wall was drilled out preserving the posterior buttress. After complete disease clearance was achieved, the posterior canal wall was reconstructed using allograft conchal cartilage. The mastoid cavity was lined by temporalis fascia harvested during the start of the surgery. BonAlive, the commercial bioglass used, is dispensed in a $10 \mathrm{cc}$ vial. Using strict sterile precautions, the granules were redistributed into six syringes of $1 \mathrm{cc}$ capacity and two syringes of 2 cc capacity. Obliteration of a moderate-to-large cavity required approximately 2cc of bioglass granules and obliteration of a small cavity required
$1 \mathrm{cc}$ of bon alive. The remaining bioglass material was stored after ETO sterilization. Thus, each fascia lined cavity was obliterated with 1 to 2 cc of bioglass granules. Following tympanoplasty, the external canal was filled with Gelfoam and a small piece of Merocel was placed. The postaural wound was then closed in layers and a drain placed for 24 hours.

Patients were followed up at 2 weeks after Merocel removal from external ear, followed by review at 1 month and then 6 months postoperatively. The primary outcome measure was the presence of a dry mastoid cavity that was free of infection and graded as per the scale developed by Merchant et al, a semiquantitative scale that is based on both patient symptoms and clinical signs. ${ }^{9}$ The scale ranges from grade 0 , which corresponds to a dry healthy cavity, to grade 3 in which there is persistent infection.

- Grade 0-No episode of otorrhea, and no pus or granulation tissue on otoscopy.

- Grade 1-One episode of otorrhea of less than 2 weeks' duration in a 3-month period or no otorrhea but a subjective feeling of wetness in the ear.

- Grade 2-More than one episode of otorrhea in a 3-month period, or an episode of otorrhea lasting more than 2 weeks, or demonstration of localized granulation tissue/pus that was promptly cured with antibiotic drops, curettage, or vinegar drops.

- Grade 3-Constant purulent otorrhea on a daily basis, or examinations showing extensive granulation tissue, or need for a revision procedure to control infection.

Secondary outcome measures included presence of postoperative complications like wound infection, posterior canal wall bulge, and residual perforation (-Fig. 1).

\section{Results}

The age of the patients studied ranged from 11 to 60 years of age. All 25 patients had cholesteatoma, while 3 of these were cases of recurrent cholesteatoma. Granulations were present in 10 patients intraoperatively. Intraoperative facial nerve dehiscence and lateral semicircular canal dehiscence were noticed in two and three patients, respectively (-Figs. 2 and $\mathbf{3}$ ).

Of the 25 cases studied, at the end of 1 month, $60 \%$ of the cases had a completely dry ear. Twenty-eight percent of patients had grade 1 and 12\% had grade 2 otorrhea as per Merchant scale. None of the patients had persistent otorrhea (-Fig. 4).
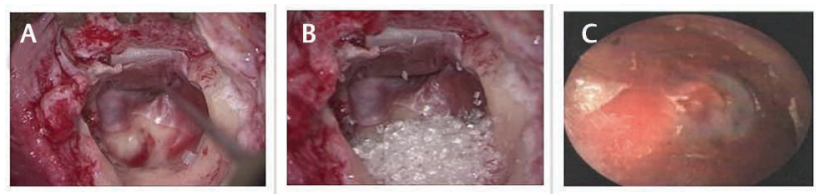

Fig. 1 (A) Intraoperative picture showing canal wall reconstruction with cartilage and temporalis graft lining mastoid cavity. (B) Intraoperative picture showing cavity obliteration with bioactive glass granules. (C) Postoperative otoendoscopy picture showing obliterated cavity after 1 month. 


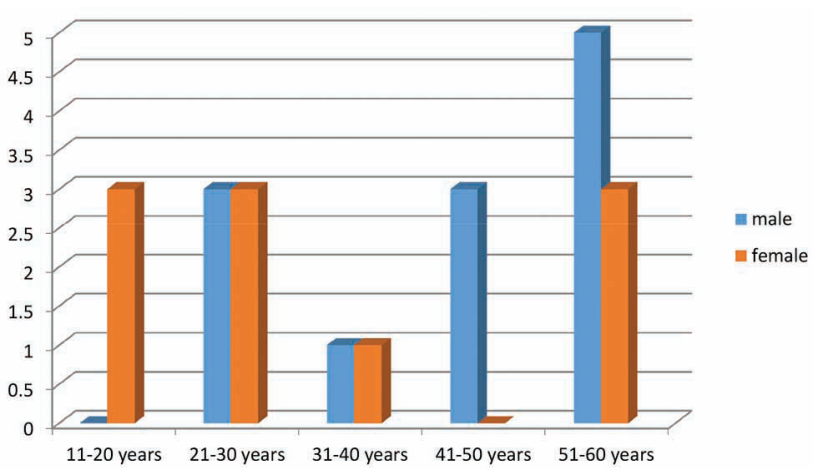

Fig. 2 Bar diagram showing the age and sex distribution of patients.

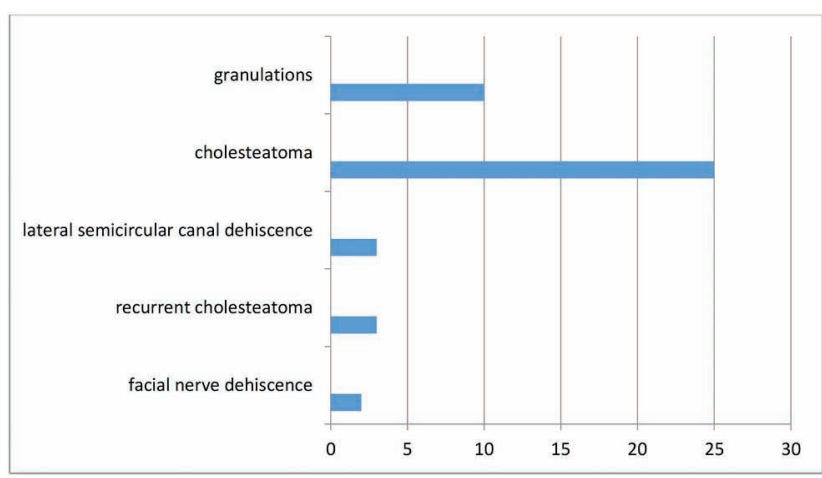

Fig. 3 Graph showing the intraoperative findings of patients.

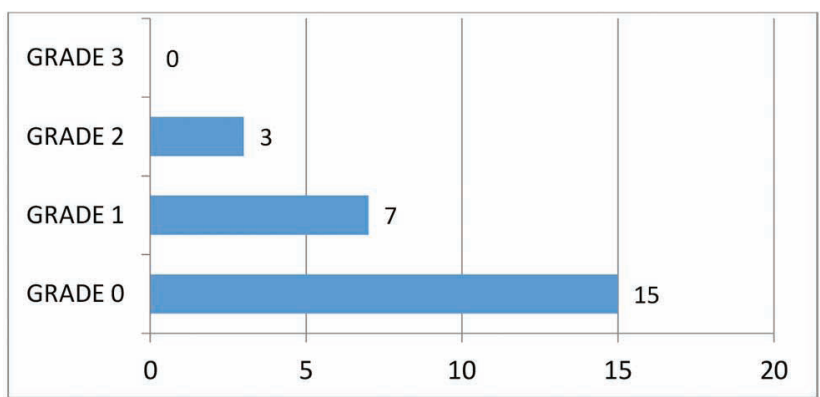

Fig. 4 Postoperative mastoid cavity status at the end of first month as per the grading by Merchant et al.

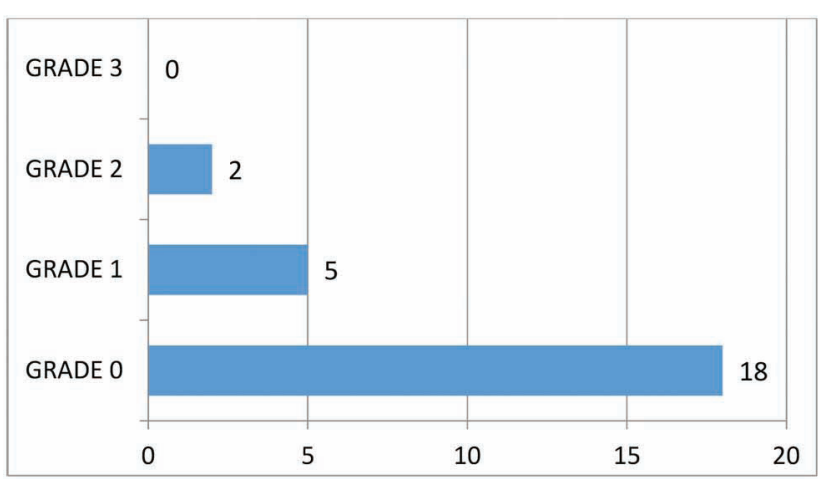

Fig. 5 Postoperative mastoid cavity status at the end of 6th month as per the grading by Merchant et al.
Primary outcome measure at the end of 6 months follow-up showed a reduction in otorrhea with $72 \%$ having a completely dry ear, while only $20 \%$ had a sensation of wetness of the ear and $8 \%$ had grade 2 otorrhea. There were no cases with grade 3 otorrhea ( - Fig. 5)

Postoperative complication of a posterior canal bulge was noted in two patients (8\%) and only one patient (4\%) had a residual perforation. None of the cases developed granulation, retraction pocket, or cavity infection.

\section{Discussion}

BAG S53P4 was used for mastoid obliteration by Sarin et al in 2012 for 25 patients with chronic otitis media. ${ }^{10}$ His technique of mastoid obliteration included a musculoperiosteal flap along with BAG granules with or without bone pate. In $96 \%$ of the patients, he obtained a dry, safe ear or only intermittent otorrhea during the median follow-up period of 34.5 months. In $92 \%$ of the patients, he was successful in obtaining a small or nonexistent cavity, thereby concluding that BAG S53P4 is a significant material for the purpose of mastoid obliteration.

The BAG S53P4 granules are composed of 53\% silicone dioxide, $23 \%$ disodium oxide, $20 \%$ calcium oxide, and $4 \%$ phosphate pentoxide. It is osteoconductive, biocompatible, bone-bonding, and nontoxic. In an aqueous environment, there is leaching of sodium ions from bioglass along with the dissolution of calcium, phosphate, and silica, thereby forming a silicon rich layer on the material, which in turn acts as a template for the precipitation of calcium phosphate which forms chemical bond with bone and soft tissue in vivo. ${ }^{11-13}$ Even previously contaminated closed bone defects were successfully obliterated with BAG when not exposed to the external environment after the procedure. ${ }^{8,14}$ The high $\mathrm{pH}$ and the nonphysiological concentration of alkali ions leached out from BAG are responsible for these effects. ${ }^{15}$ Further to this, it also exerts an inhibitory effect on the colonization of Pseudomonas aeruginosa. Till date there has not been any clear documentation of BAG-associated infections observed in clinical studies.

In this study, we obtained results comparable with literature obtaining a completely dry ear in $60 \%$ cases at the end of 1 month and 72\% at the end of 6 months. None of the patients developed grade 3 otorrhea. We can compare our study results with studies of Stoor et al and Sarin et al where obliteration of cavities was done with BAG S53P4..$^{10,16}$ In the area of posterior canal wall, we used temporalis fascia as a cover for bioactive granules thus completely confining the BAG granules to the fascial sac. Only one patient presented after surgery with prolonged otorrhea, which implied a possibility of a persistent active disease, which was not removed during surgery. However, the otorrhea ceased with prolonged oral and topical antibiotic treatment and became dry during the following 6 months. A similar case was also reported in Stoor et al study. Postoperative complication was seen in only three cases, where a posterior canal bulge was noted in 
two patients ( $8 \%$ ) and only one patient (4\%) had a residual perforation. None of the cases developed granulation, retraction pocket, or cavity infection. Thus, BAG S53P4 appears to have better osseointegrating properties compared with its predecessors. The antimicrobial and angiogenesis stimulating properties help in long-term cavity obliteration without undergoing ischemic atrophy.

The new technique of application of bioglass within a fascia-lined sac has numerous advantages. Being contained within the mastoid cavity, it stimulates osteogenesis within the cavity only, keeping sensitive structures such as the ossicular chain, inner ear, and facial nerve protected from the high alkaline $\mathrm{pH}$ of the material making it safe to use in cases with fractured stapes footplate, dehiscent facial canal, or lateral semicircular canal dehiscence/fistula. Additionally, this technique prevents development of external canal dehiscence. Redistribution of the material into 2cc aliquots makes its more economical and cost-effective to be used in all cases. The high alkaline $\mathrm{pH}$ prevents bacterial growth and subsequent infection of the cavity.

Healing of the cavity has been noted to be better with application of bioglass granules within a fascial covering than when placed directly into mastoid cavity in contact with bare bone. Persistent active disease within the mastoid cavity has been considered to be a relative contraindication for obliteration with bioglass. We here present a set of recommendations to the use of bioglass based on our experience:

1. A well-saucerized mastoid cavity.

2. Removing all granulations and effusing mucosa and ensuring cholesteatoma clearance is complete.

3. Reconstructing canal wall with overlapping pieces of cartilage.

4. Filling up of external canal with Gelfoam/Merocel for at least 2 weeks to stent canal and prevent posterior canal bulging.

5. Filling up cavity up to level of cortical bone and insertion of a drain into the wound is a must.

\section{Conclusion}

The clinical performance, safety, and efficacy of BAG with regard to osteostimulation were proven in addition to its antimicrobial property. The ease of application of the granules within a facial sac thus protecting delicate structures along with the cost-effectiveness achieved by redistribution of the total volume in a hassle-free manner makes this a near-ideal obliteration material. The procedure can be performed under local anesthesia, along with reduction in the intraoperative time and hospital stay. However, follow-up for longer periods and multicenter studies including a broader number of cases need to be initiated to reassure the outcome of our experience.

\section{Ethical Approval}

This study was performed in line with the principles of the Declaration of Helsinki. Approval was granted by the institution ethics committee.

\section{Consent to Participate}

Informed consent was obtained from all individual participants included in the study.

\section{Consent for Publication}

The participants have consented to the submission of the case details for publication.

\section{Availability of Data and Material}

Data transparency has been maintained.

\section{Authors' Contributions}

All authors made substantial contribution to this article and all the authors have approved the version to be published.

\section{Funding}

No funds, grants, or other support was received.

\section{Conflicts of Interest}

The authors have no conflicts of interest to declare that are relevant to the content of this article.

\section{References}

1 Mosher HP. A method of filling the excavated mastoid with a flap from the back of the auricle. Laryngoscope 1911;21:1158-1163

2 Mehta RP, Harris JP. Mastoid obliteration. Otolaryngol Clin North Am 2006;39(6):1129-1142

3 Lambert PR. Mastoidectomy. Chapter 142: Cummings Otolaryngology Head and Neck Surgery. 5th edition. Philadelphia, PA: Mosby Elsevier; 2010:2009-2016

4 Munukka E, Leppäranta O, Korkeamäki M, et al. Bactericidal effects of bioactive glasses on clinically important aerobic bacteria. J Mater Sci Mater Med 2008;19(1):27-32

5 Peltola M, Aitasalo K, Suonpää J, Varpula M, Yli-Urpo A. Bioactive glass S53P4 in frontal sinus obliteration: a long-term clinical experience. Head Neck 2006;28(9):834-841

6 Suominen E, Kinnunen J. Bioactive glass granules and plates in the reconstruction of defects of the facial bones. Scand J Plast Reconstr Surg Hand Surg 1996;30(4):281-289

7 Turunen T, Peltola J, Yli-Urpo A, Happonen RP. Bioactive glass granules as a bone adjunctive material in maxillary sinus floor augmentation. Clin Oral Implants Res 2004;15(2):135-141

8 Aitasalo KMJ, Peltola MJ. Bioactive glass hydroxyapatite in fronto-orbital defect reconstruction. Plast Reconstr Surg 2007;120(7):1963-1972

9 Merchant SN, Wang P, Jang CH, et al. Efficacy of tympanomastoid surgery for control of infection in active chronic otitis media. Laryngoscope 1997;107(7):872-877

10 Sarin J, Grénman R, Aitasalo K, Pulkkinen J. Bioactive glass S53P4 in mastoid obliteration surgery for chronic otitis media and cerebrospinal fluid leakage. Ann Otol Rhinol Laryngol 2012;121(9):563-569

11 Hench LL, Paschall HA. Direct chemical bond of bioactive glass-ceramic materials to bone and muscle. J Biomed Mater Res 1973;7(3):25-42

12 Hench LL, Splinter RJ, Allen WC, Greenlee TK. Bonding mechanism at the interface of ceramic prosthetic materials. J Biomed Mater Res 1971;5:117-141

13 Häkkinen L, Yli-Urpo A, Heino J, Larjava H. Attachment and spreading of human gingival fibroblasts on potentially bioactive glasses in vitro. J Biomed Mater Res 1988;22(11):1043-1059

14 Peltola M, Suonpää J, Aitasalo K, Varpula M, Yli-Urpo A, Happonen R-P. Obliteration of the frontal sinus cavity with bioactive glass. Head Neck 1998;20(4):315-319 
15 Stoor P, Söderling E, Salonen JI. Antibacterial effects of a bioactive glass paste on oral microorganisms. Acta Odontol Scand 1998;56(3):161-165
16 Stoor P, Pulkkinen J, Grénman R. Bioactive glass S53P4 in the filling of cavities in the mastoid cell area in surgery for chronic otitis media. Ann Otol Rhinol Laryngol 2010;119(6):377-382 